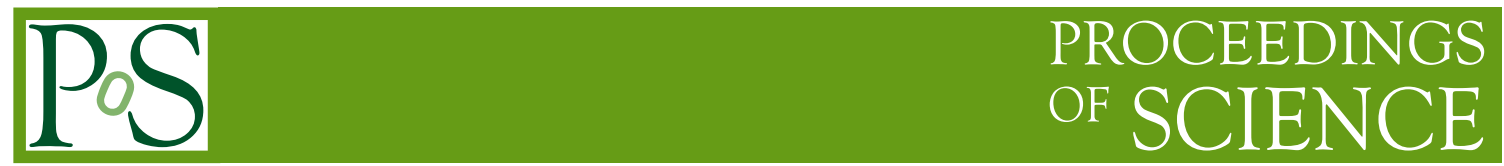

\title{
Differential jet cross sections at the CMS experiment
}

\author{
Radek Žlebčík* \\ on behalf of the CMS Collaboration \\ DESY \\ E-mail: radek.zlebcik@desy.de
}

We present measurements of differential jet cross sections over a wide range in transverse momenta from inclusive jets to multi-jet final states. Studies on the impact that these measurements have on the determination of the strong coupling $\alpha_{S}$ as well as on parton density functions are reported. Several LO and NLO MC generator matched with the parton shower were tested against the CMS data.

XXVII International Workshop on Deep-Inelastic Scattering and Related Subjects - DIS2019 8-12 April, 2019

Torino, Italy

${ }^{*}$ Speaker. 


\section{Introduction}

In high-energy collisions, narrow collimated clusters of the produced particles are observed, so-called jets. Within the framework of the QCD improved parton model, jets are related to partons produced via partonic hard sub-process, which then hadronize to "jets" of stable particles observed in the detector. In case of proton-proton ( $p p$ ) collisions, the cross section is expressed as a convolution of the universal parton distribution functions (PDFs) and the process-dependent partonic sub-process cross section, which can be calculated within perturbative QCD.

Jets reconstructed from partons of the hard sub-process can be directly related to the particlelevel jets. However, to obtain a more realistic description of the data, the effects of parton showering, multi-parton interactions and hadronization must be taken into account. These processes are typically simulated by MC generators and depend on several phenomenological assumptions. Studying the jet production allows probing all these effects, especially the calculations of the hard process cross section (for dijets available up to next-to-next-to-leading order in $\alpha_{S}$ ) and the parton shower effects, which are crucial for more exclusive observables, infrared unsafe observables, or for high parton multiplicities. In addition, since the event rate is driven by the parton densities in the proton, the jet production provides a strong constraint to the partonic structure of the proton.

The CMS [1], together with ATLAS, is a general-purpose detector at the Large Hadron Collider (LHC) at CERN. In this text we present several studies of processes involving jet production in $p p$ collisions at centre-of-mass energies $\sqrt{s}=8$ and $13 \mathrm{TeV}$ based on data from Run 1 and Run 2, collected between the years 2012 and 2016.

In the experiment, jets are clustered from the, so-called, particle flow candidates, obtained by Particle Flow (PF) algorithm [2], which combines information from the CMS sub-detectors. Most importantly, both tracks and clusters are used to reconstruct the energy of the hadrons. Inclusion of the tracker information substantially improves the transverse momentum $\left(p_{\mathrm{T}}\right)$ resolution at low and intermediate $p_{\mathrm{T}}$. The CMS tracker covers the pseudorapidity region $|\eta|<2.5$, whereas the calorimeter extends up to $|\eta|<4$.7. Consequently, in the central region $(|\eta|<2.5)$, the jets are reconstructed with much better energy resolution since both tracker and calorimeter are involved.

The jets are defined by the anti- $k_{\mathrm{T}}$ algorithm [3] run in the laboratory frame (implemented in the FASTJET package [4]). The jet algorithm is infrared safe and boost invariant along the $z$-axis. From the experimental point of view, the anti- $k_{\mathrm{T}}$ jet algorithm keeps the jets circular in $(y, \phi)$ plane with a radius roughly equal to the jet size-parameter $R$. This property is important to control the Pile-Up induced background from other interactions under control.

All presented distributions are corrected for detector effects to particle-level using an unfolding technique. The particle-level by definition includes stable particles with the lifetime $\tau$ satisfying $\tau c>1 \mathrm{~cm}$, excluding neutrinos. Therefore, the distributions can be directly compared to the MC predictions or to fixed-order NLO predictions corrected for electroweak and non-perturbative QCD effects.

\section{Inclusive jet production}

The inclusive jet production is a flag-ship Standard Model measurement allowing to test the Standard Model predictions at the highest scales and probe the proton structure at the smallest 
distances $\sim 1 \mathrm{am}$. The jet cross section is measured double-differentially in bins of jet transverse momentum $p_{\mathrm{T}}$ and rapidity $y$. In Run 1 the cross sections were measured with beam energies of 7 and $8 \mathrm{TeV}$, from Run 2 only $13 \mathrm{TeV}$ analysis of early 2015 data exists so far. The $8 \mathrm{TeV}$ analysis [5] includes jets with $R=0.7$, wheres in the $13 \mathrm{TeV}$ analysis [6] the jets for $R=0.4$ and $R=0.7$ were measured. Note, that the $8 \mathrm{TeV}$ analysis has much higher luminosity, up to $19.7 \mathrm{fb}^{-1}$, compared to the $13 \mathrm{TeV}$ measurement which is based on data with $\sim 71 \mathrm{pb}^{-1}$.

The measured differential distributions at 8 and $13 \mathrm{TeV}$ centre-of-mass energies can be seen in Fig. 1. In the low $p_{\mathrm{T}}$ region $p_{\mathrm{T}}<74 \mathrm{GeV}$ of $8 \mathrm{TeV}$ measurement, where the cross section is
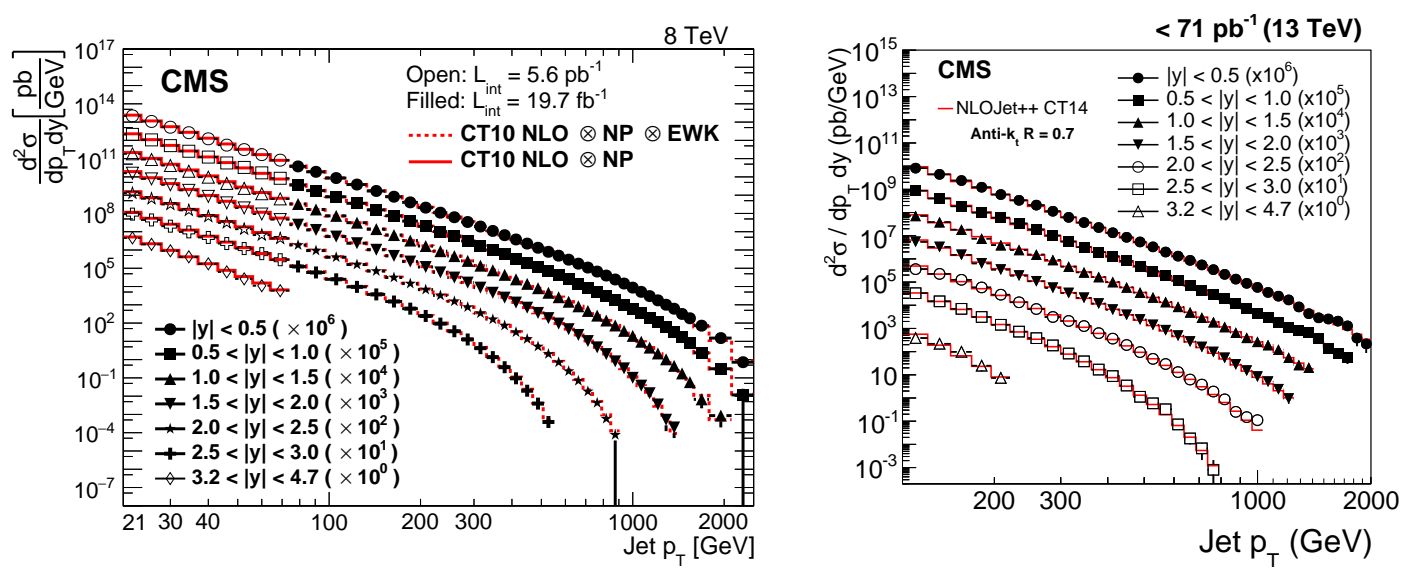

Figure 1: The double-differential inclusive jet cross sections in bins of absolute value of $|y|$ and $p_{\mathrm{T}}$ for $p p$ collisions at $8 \mathrm{TeV}$ [5] (left) and $13 \mathrm{TeV}$ [6] (right). The data points are accompanied with fixed order NLO predictions based on CT10 or CT14 PDF.

large, a dedicated low-Pile-Up sample with luminosity $5.6 \mathrm{pb}^{-1}$ was studied to reduce the Pile-Up uncertainty which is the dominant source for standard high-Pile-Up runs in this region. The data extend up to $|y|=5.0$ in the low $p_{\mathrm{T}}$ region and to $|y|=3.0$ for $p_{\mathrm{T}}>74 \mathrm{GeV}$.

Higher statistics and a better understanding of the systematic uncertainties of the $8 \mathrm{TeV}$ measurement (compared to the analysis of early $13 \mathrm{TeV}$ data) allow for QCD fits. We performed a combined NLO QCD fit of the $8 \mathrm{TeV}$ jet data with $p_{\mathrm{T}}>74 \mathrm{GeV}$ together with the HERA DIS measurements. In the left plot of Fig. 2 it can be seen that the inclusion of the jet data substantially reduces the gluon PDF uncertainty at high scales and at high- $x$. In addition the strong coupling $\alpha_{S}\left(M_{Z}\right)$ was extracted. The value $\alpha_{S}^{\mathrm{NLO}}\left(M_{Z}\right)=0.1164_{-0.0043}^{+0.0060}$, with error dominated by the QCD scale uncertainty, agrees with the world average value $0.1181 \pm 0.0011$ [7]. To study the $\alpha_{S}$ running, the QCD analysis was also performed independently for seven intervals of the jet $p_{\mathrm{T}}$ (Fig. 2 right). In this way, the $\alpha_{S}$ running, driven by the renormalization group equation, was experimentally tested for scales between $86 \mathrm{GeV}$ and $1.5 \mathrm{TeV}$.

\section{Triple differential dijet cross section}

Measurements of the dijet events allow for additional constraints of the PDFs and $\alpha_{S}$ and the MC models. The cross sections were measured triple-differentialy, in the average $p_{\mathrm{T}}$ of the leading and sub-leading jets $p_{T, \text { avg }}=\frac{1}{2}\left(p_{T, 1}+p_{T, 2}\right)$, in the mean rapidity of the dijet system $y_{b}=\frac{1}{2}\left(y_{1}+y_{2}\right)$, 

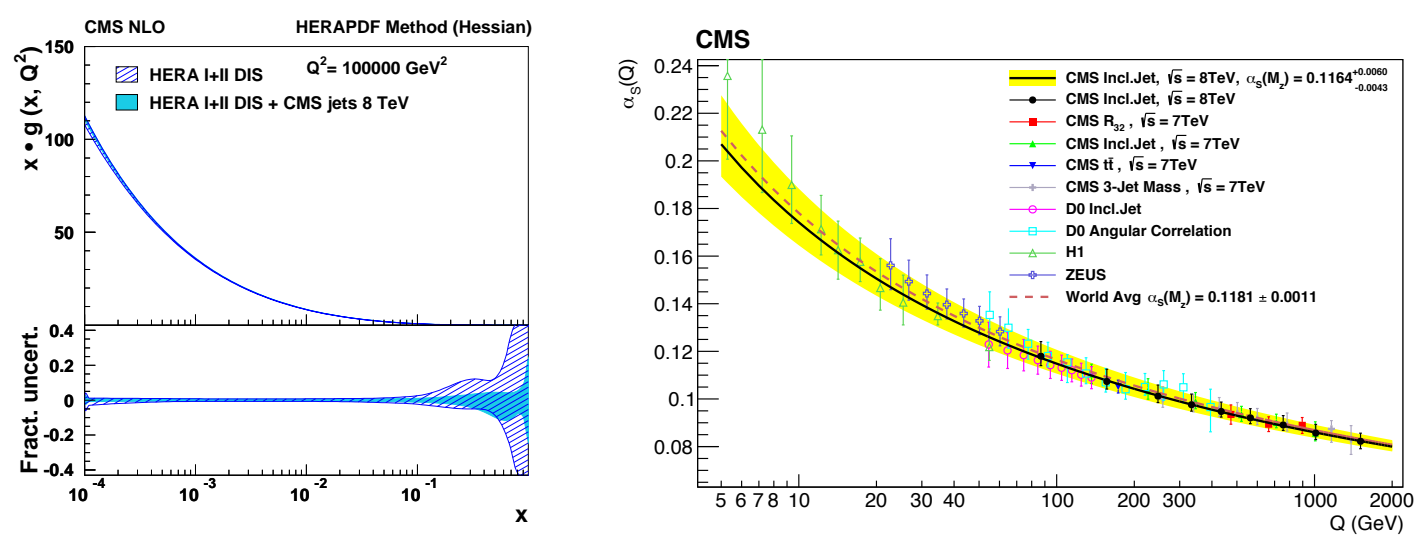

Figure 2: The left plot demonstrates the impact of the $8 \mathrm{TeV}$ jet data [5] on the gluon PDF uncertainty at scale $Q=316 \mathrm{GeV}$, where the hatched band represents the uncertainty when only HERA data are fitted, whereas the solid band corresponds to the fit which includes jet data as well. The plot on the right hand side shows running of $\alpha_{S}$ as measured by various experiments. The CMS $8 \mathrm{TeV}$ inclusive jet data are plotted as black bullets.

and in the jet rapidity in the boosted dijet frame, $y^{*}=\frac{1}{2}\left|y_{1}-y_{2}\right|$. The data analysis is based on the $8 \mathrm{TeV}$ data with integrated luminosity $19.7 \mathrm{fb}^{-1}$ [8]. Jets are defined by the anti- $k_{\mathrm{T}}$ algorithm with $R=0.7$ must have $p_{\mathrm{T}}>50 \mathrm{GeV}$. In total, the $p_{T \text {,avg }}$ spectrum was binned to 6 rapidity regions in $y^{*}$ and $y_{b}$ as can be seen in Fig. 3. The data are mostly described by the fixed order NLO
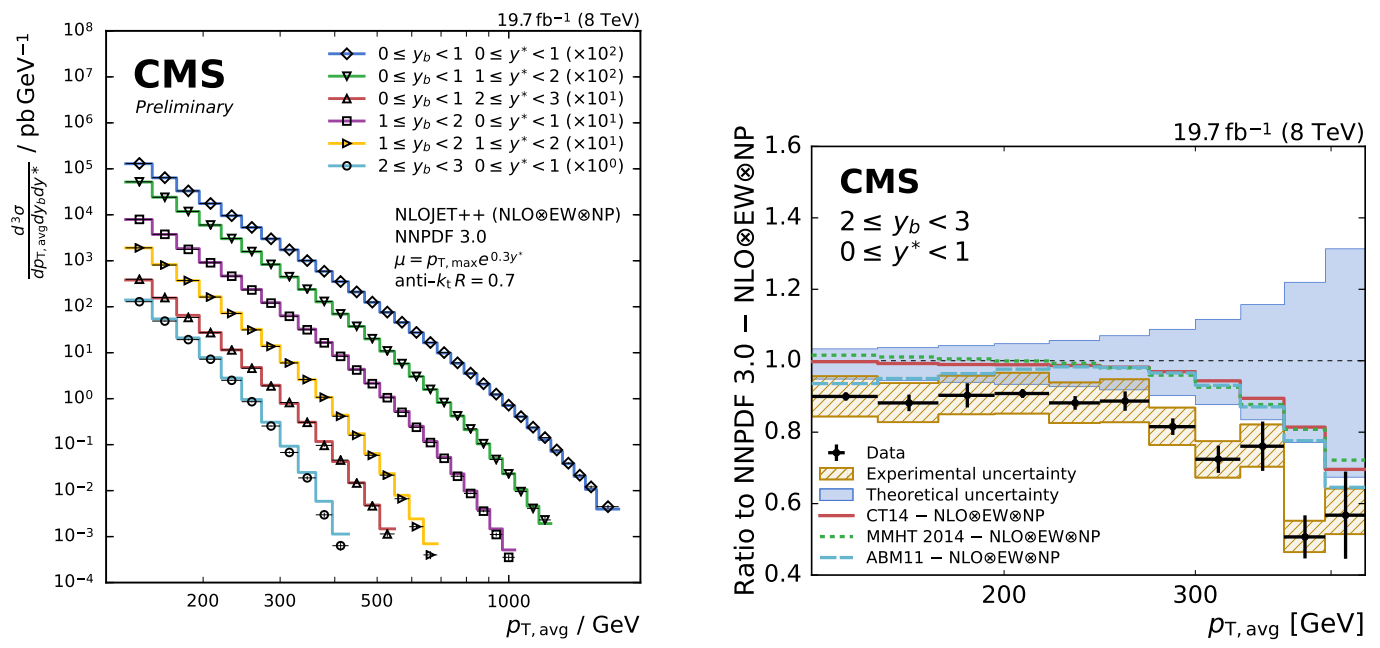

Figure 3: Triple-differential dijet cross sections in six bins of $y^{*}$ and $y_{b}$ are presented [8]. The data are indicated by different markers for each bin. The NLO theoretical predictions corrected for electroweak and non-perturbative effects are depicted by solid lines. The right plot shows the ratio with respect to the NLO prediction based on NNPDF 3.0 PDF. The predictions based on CT14, MMHT 2014 and ABM11 PDFs are also included.

predictions corrected for NP and EW effects based on NNPDF 3.0 NLO parton densities. Only in the boosted region, $2<y_{b}<3$ and $0<y^{*}<1$, the predictions overestimate the data at higher $p_{\mathrm{T}}$. This rapidity region is dominated by the quark-gluon scattering, where quark has high $x$ and gluon 
small $x$. However, the ratio plot shows that predictions in this region have high PDF uncertainty, much higher than the uncertainty of the data. Therefore, the measured triple-differential data allow to better constrain parton densities.

A QCD fit based on these CMS dijet measurement and HERA DIS data sets was performed, similarly as in the inclusive jet analysis. It was observed that the dijet data are able to better constrain gloun and $u_{v}$ and $d_{v}$ PDFs at high $x$ and high scales, compared to HERA data alone. The fitted $\alpha_{S}$ value, $\alpha_{S}^{\mathrm{NLO}}\left(M_{Z}\right)=0.1199_{-0.0020}^{+0.0035}$, has uncertainties dominated by the QCD scale and are slightly lower than for the inclusive jet measurement.

\section{Azimuthal correlations}

In the framework of fixed order pQCD calculations, the partons in $2 \rightarrow 2$ process are produced exactly back-to-back, in other words the difference between azimuthal angle of the leading and sub-leading jet in $p_{\mathrm{T}}, \Delta \phi_{1,2}=\left|\phi^{\mathrm{jet} 1}-\phi^{\mathrm{jet} 2}\right|=\pi$. Adding an extra leg to the final state $(2 \rightarrow 3)$ allows to produce values above $\Delta \phi_{1,2}^{\min }=\frac{2}{3} \pi$, in general $\Delta \phi_{1,2}^{\min }=\frac{2 \pi}{n}$, where $n$ is number of partons in the final state. The phase space bellow $\Delta \phi_{1,2}^{\min }$ cannot be filled by the fixed order predictions.

The angular differences close to $\pi$ are problematic as well, since the fixed order tree-level predictions, for 3 or more partons in the final state, diverge in this region. At NLO the positive (real) divergent part is canceled by the negative (virtual) contribution lying exactly at $\Delta \phi_{1,2}=\pi$, but the distribution is still ill-defined in the $\Delta \phi_{1,2} \sim \pi$ region.

To conclude, the fixed order calculations are expected to perform well in the $\Delta \phi_{1,2}$ region safely above $\Delta \phi_{1,2}^{\min }$ and safely below $\pi$. To describe the data outside this region, the resummation made implicitly by the parton shower matched to the hard partonic sub-process is needed, while the pure fixed order predictions fail.

The CMS collaboration measured $\Delta \phi_{1,2}$ distribution on a 2016 data with an integrated luminosity $35.9 \mathrm{fb}^{-1}$. The jets selected by anti- $k_{\mathrm{T}}$ algorithm with $R=0.4$ were required to have $p_{\mathrm{T}}$ above $100 \mathrm{GeV}$. The normalized $\Delta \phi_{1,2}$ spectra were measured in several bins of $p_{\mathrm{T}}$ of the leading jet. The analysis was performed with 2,3 or 4 jets required in the final state, for simplicity, in Fig. 4 only data/MC distributions for 2 jets in the final state are shown. Comparison with the leading order generators (left plot) shows that MADGRAPH+PYTHIA8 (tune CUETP8M1) performs the best and this is also the case for 3-jet and 4-jet scenario. In MADGRAPH the $2 \rightarrow 2,2 \rightarrow 3$ and $2 \rightarrow 4$ processes are included in the matrix element and matched to the PYTHIA 8 shower using the MLM matching scheme. The pure PythiA 8 and HeRWIG++ include only $2 \rightarrow 2$ hard process. The right plot in Fig. 4 compares data with NLO MC generators. It can be seen that HERwIG7 which includes $2 \rightarrow 2$ at NLO and $2 \rightarrow 3$ at LO and is using MC@ NLO technique performs best. Surprisingly, the POWHEG+3J + PYTHIA8 (tune CUETP8M1) having $2 \rightarrow 2$ and $2 \rightarrow 3$ at NLO and $2 \rightarrow 4$ at LO provides worse data compared to LO MADGRAPH shown on the left plot.

The back-to-back region of $\Delta \phi_{1,2}$, i.e. $\Delta \phi_{1,2}=170^{\circ} \ldots 180^{\circ}$, which is very sensitive to extra soft radiation and Sudakov resummation over large range of scales was studied separately in [10] (Fig. 5). The analysed data set and jet definition was identical as in the previous measurement. The angular jet resolution allows measuring with bins of size $1^{\circ}$. It can be seen (Fig. 5) that with increasing transverse momentum of the leading jet $p_{T}^{\max }$ the 2-jet $\Delta \phi_{1,2}$ is more and more peaked at $\Delta \phi_{1,2}=180^{\circ}$. However, when an extra jet is required the leading jets are much more decorrelated. 

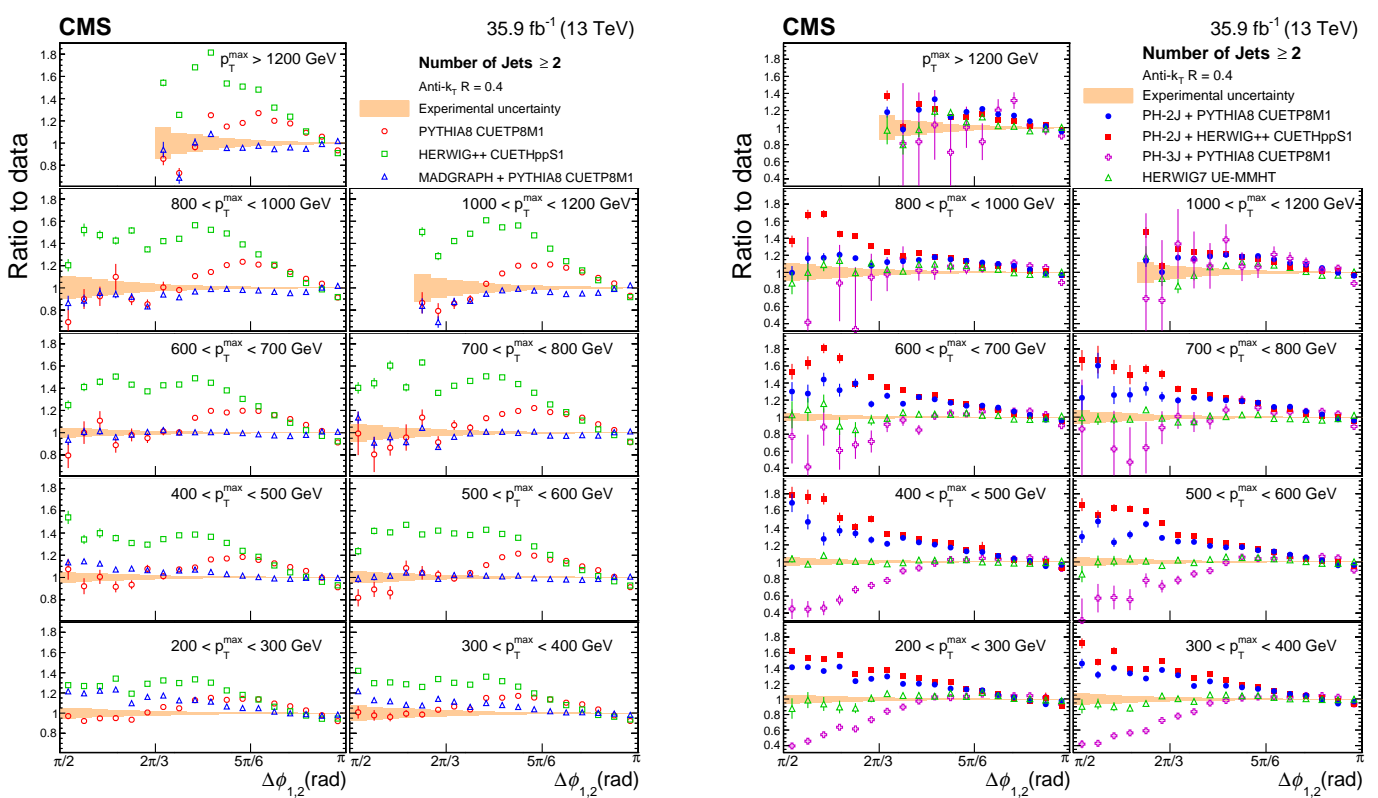

Figure 4: The ratio of the normalized distributions $\frac{1}{\sigma} \frac{d \sigma}{d \Delta \phi_{1,2}}$ to the distribution in data as measured for 2-jet scenario [9]. The spectra are always normalized to the total dijet cross section in given range of $p_{T, 1}$ and $p_{T, 2}$. The overall experimental uncertainties are plotted by the orange band. The statistical uncertainties of the MC predictions are depicted by the vertical error bars.

The data were compared to several LO and NLO predictions, for example the Fig. 6 shows the comparison to the Pythia8, HERWIG++ and MADGRAPH+Pythia8, i.e. the same generators as were shown in the left plot of Fig. 4. Apparently MADGraPH+Pythia8 still provides best description for 2-jet scenario but deviates from data for 3-jet selection in the back-to-back region.

\section{References}

[1] CMS collaboration, The CMS Experiment at the CERN LHC, JINST 3 (2008) S08004.

[2] CMS collaboration, Particle-flow reconstruction and global event description with the CMS detector, JINST 12 (2017) P10003 [1706. 04 965].

[3] M. Cacciari, G. P. Salam and G. Soyez, The anti- $k_{t}$ jet clustering algorithm, JHEP 04 (2008) 063 [0802.1189].

[4] M. Cacciari, G. P. Salam and G. Soyez, FastJet User Manual, Eur. Phys. J. C72 (2012) 1896 [1111.6097].

[5] CMS collaboration, Measurement and QCD analysis of double-differential inclusive jet cross sections in pp collisions at $\sqrt{s}=8 \mathrm{TeV}$ and cross section ratios to 2.76 and $7 \mathrm{TeV}, \mathrm{JHEP} 03$ (2017) 156 [1609.05331].

[6] CMS collaboration, Measurement of the double-differential inclusive jet cross section in protonâĂŞproton collisions at $\sqrt{s}=13$ TeV, Eur. Phys. J. C76 (2016) 451 [1605. 04436 ].

[7] Particle Data Group collaboration, Review of Particle Physics, Phys. Rev. D98 (2018) 030001. 

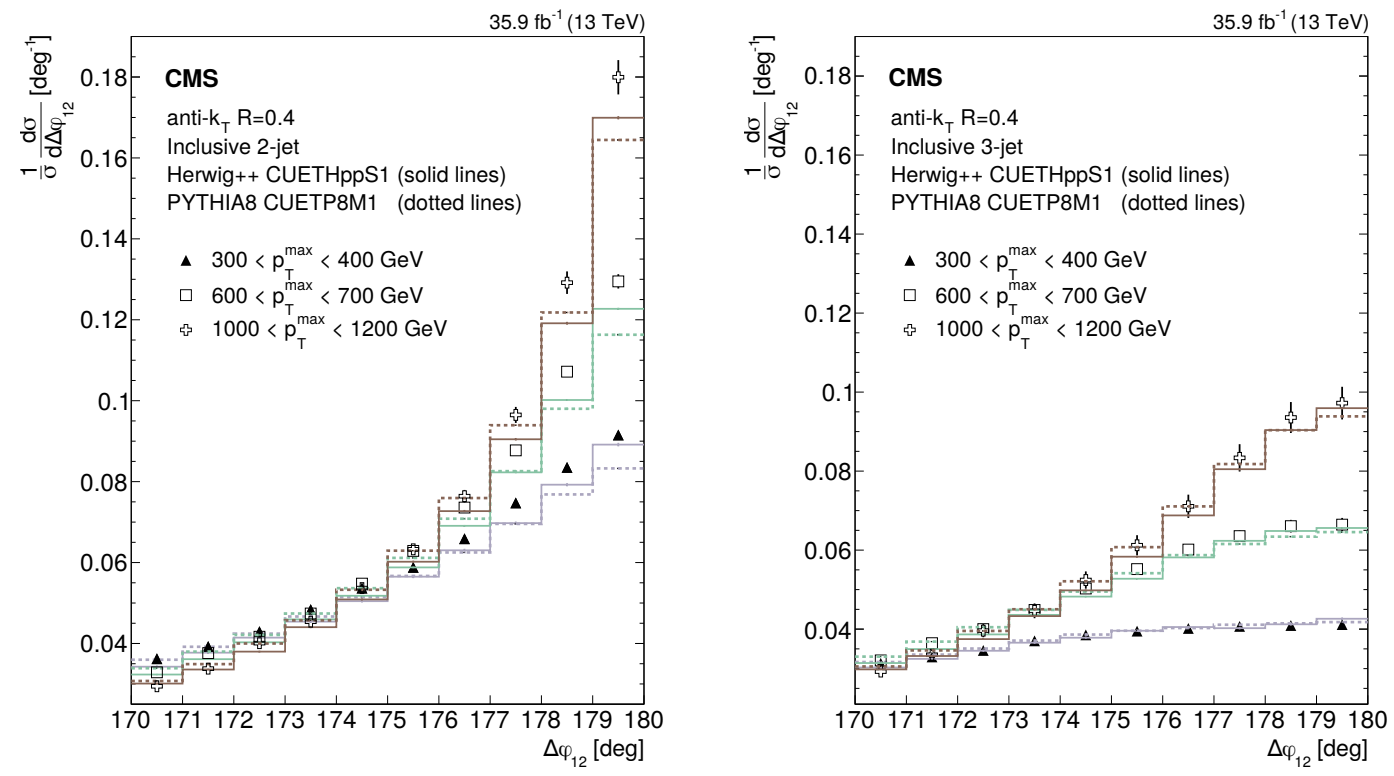

Figure 5: The normalized $\Delta \phi_{1,2}$ distribution measured for 2-jet and 3-jet scenario in the back-to-back region for several intervals of the leading jet $p_{\mathrm{T}}[10]$. In addition to the data points, the predictions from HERWIG++ and PYTHIA8 MC generators are plotted.
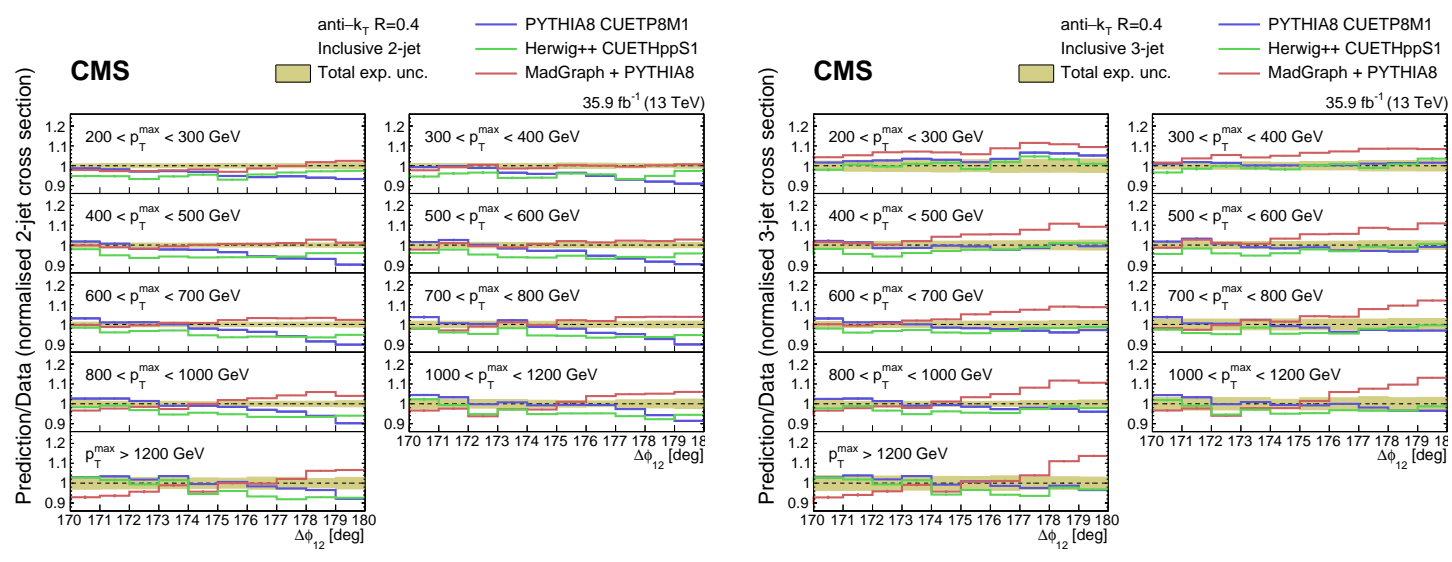

Figure 6: The ratio of normalized $\Delta \phi_{1,2}$ distribution measured for 2-jet and 3-jet scenario in the back-toback region for several intervals of the leading jet $p_{\mathrm{T}}[10]$ to the data. The overall experimental uncertainty is depicted as an orange band. The predictions from HERWIG++, Pythia8 and MADGRAPH+PyThIA 8 generators are plotted.

[8] CMS collaboration, Measurement of the triple-differential dijet cross section in proton-proton collisions at $\sqrt{s}=8 \mathrm{TeV}$ and constraints on parton distribution functions, Eur. Phys. J. C77 (2017) $746[1705.02628]$.

[9] CMS collaboration, Azimuthal correlations for inclusive 2-jet, 3-jet, and 4-jet events in pp collisions at $\sqrt{s}=13$ TeV, Eur. Phys. J. C78 (2018) 566 [1712.05471].

[10] CMS collaboration, Azimuthal separation in nearly back-to-back jet topologies in inclusive 2-and 3-jet events in pp collisions at $\sqrt{s}=13 \mathrm{TeV}, 1902.04374$. 